\title{
Against the Tide: Psychiatric Oversight and Consultation in Child Welfare Systems May Help Reduce Inappropriate Polypharmacy
}

\author{
Paul Rao, MD \\ Connecticut Department of Children and Families (DCF) \\ Yale University School of Medicine \\ paul.rao@yale.edu
}

Objective: To show that the establishment of a psychiatric authorization and consultation division within the child welfare system may help reduce antipsychotic prescribing and polypharmacy.

Background: In the US, youth in foster care, for whom the effects of developmental trauma are often the sine qua non, are prescribed polypharmacy regimens, including concurrent antipsychotics, at rates above the national average ${ }^{1-3}$. In response, federal agencies have mandated that states develop systems of oversight of psychotropic medications for youth in foster care who are vulnerable to inappropriate polypharmacy and associated adverse health consequences $^{45}$. The Connecticut state legislature mandated that its child welfare agency (DCF) establish a division to provide informed consent and ongoing monitoring for psychiatric medications for youth for whom it is legal guardian. This unit, the Centralized Medication Consent Unit (CMCU), is staffed by child psychiatrists and psychiatric nurse practitioners, and its psychiatrists provide consultation to child welfare teams and community providers. The CMCU began tracking data on the total number of psychotropic medications in 2010 , and on concurrent antipsychotic prescribing in 2012.

Methods: For youth under age 18 for whom the state of CT DCF has been adjudicated legal guardian, and for whom psychiatric medication is requested, the CMCU reviews whether the request is clinically appropriate and gives written informed consent to the prescriber. Figure 1 shows the number of requests per year, unique youth these requests represent, and the total number of children in DCF care. Beginning in 2013, requests for a $5^{\text {th }}$ psychotropic medicine or concomitant antipsychotic trigger an automatic phone consultation to the prescriber to review the case. The number of unique youth consented for 5 or more concurrent psychotropic medications for psychiatric indications and for two or more concurrent standing antipsychotics, not counting cross tapers, emergency, or PRN use, were tallied from a centralized database.

Figure 1: Total Number of Requests per Year

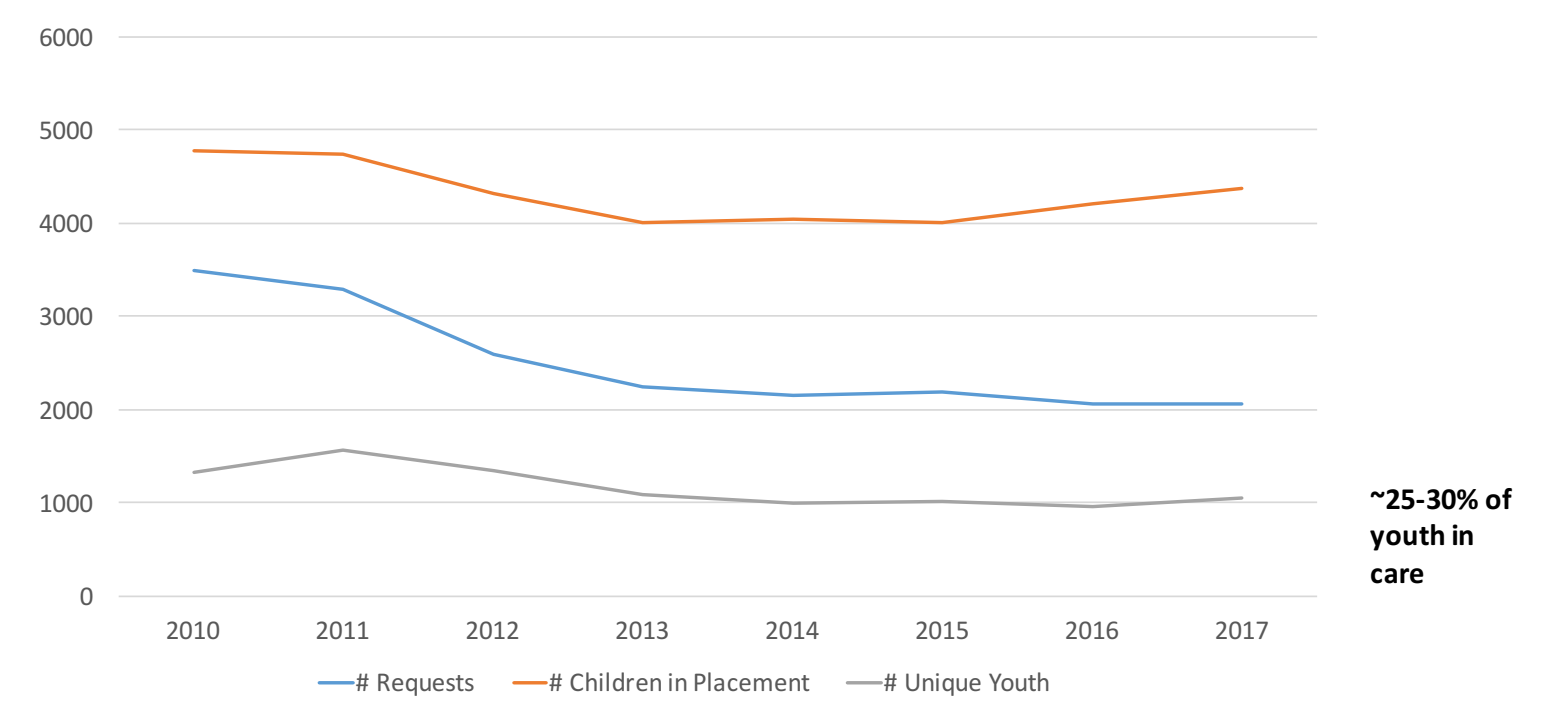

Results: Figure 2 shows that between 2010 and 2017 there was an $86 \%$ reduction in the number of DCF committed youth approved for 5 or more psychotropic medications, and no youth have been approved for more than 5 since 2014 . Figure 3 shows that between 2012 and 2017 there was a 94\% reduction in DCF committed youth approved for 2 concurrent antipsychotics.

Significance and Caveats: Authorization by a psychiatric team that also acts in loco parentis as part of the child welfare agency may provide a level of oversight that can help reduce irrational prescribing. The capacity to provide direct phone consultation to prescribers can mitigate potential conflict around the process and raise awareness about the challenges of prescribing judiciously for foster youth. Limitations of this report include: the inability to establish causality; and the independence of the DCF database from insurance and pharmacy databases, limiting the ability to more accurately establish duration of treatment with different medicines.

Figure 2: Youth on 2 concurrent antipsychotics

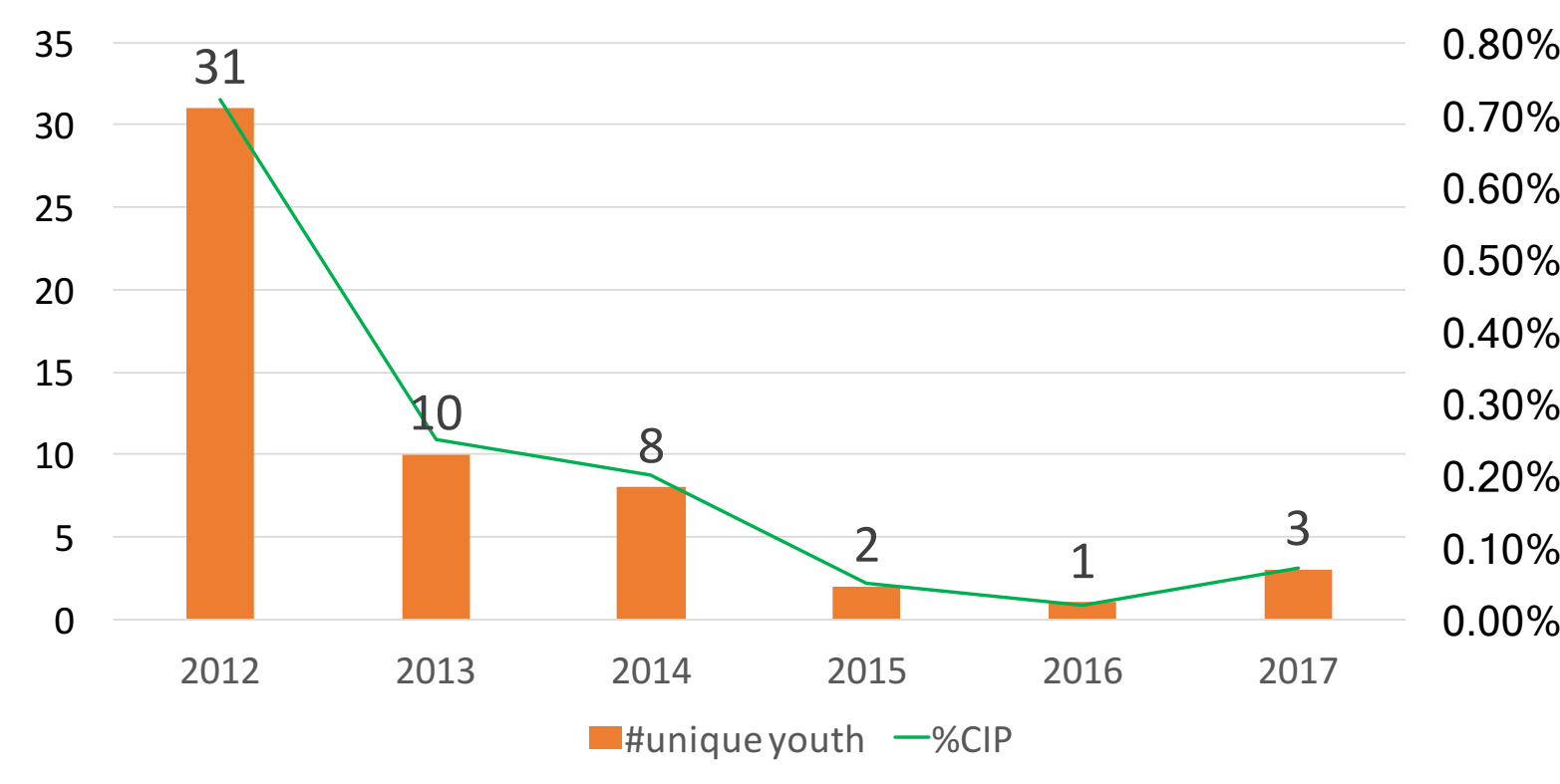

Figure 3: Youth on $\geq 5$ psychotropic medications

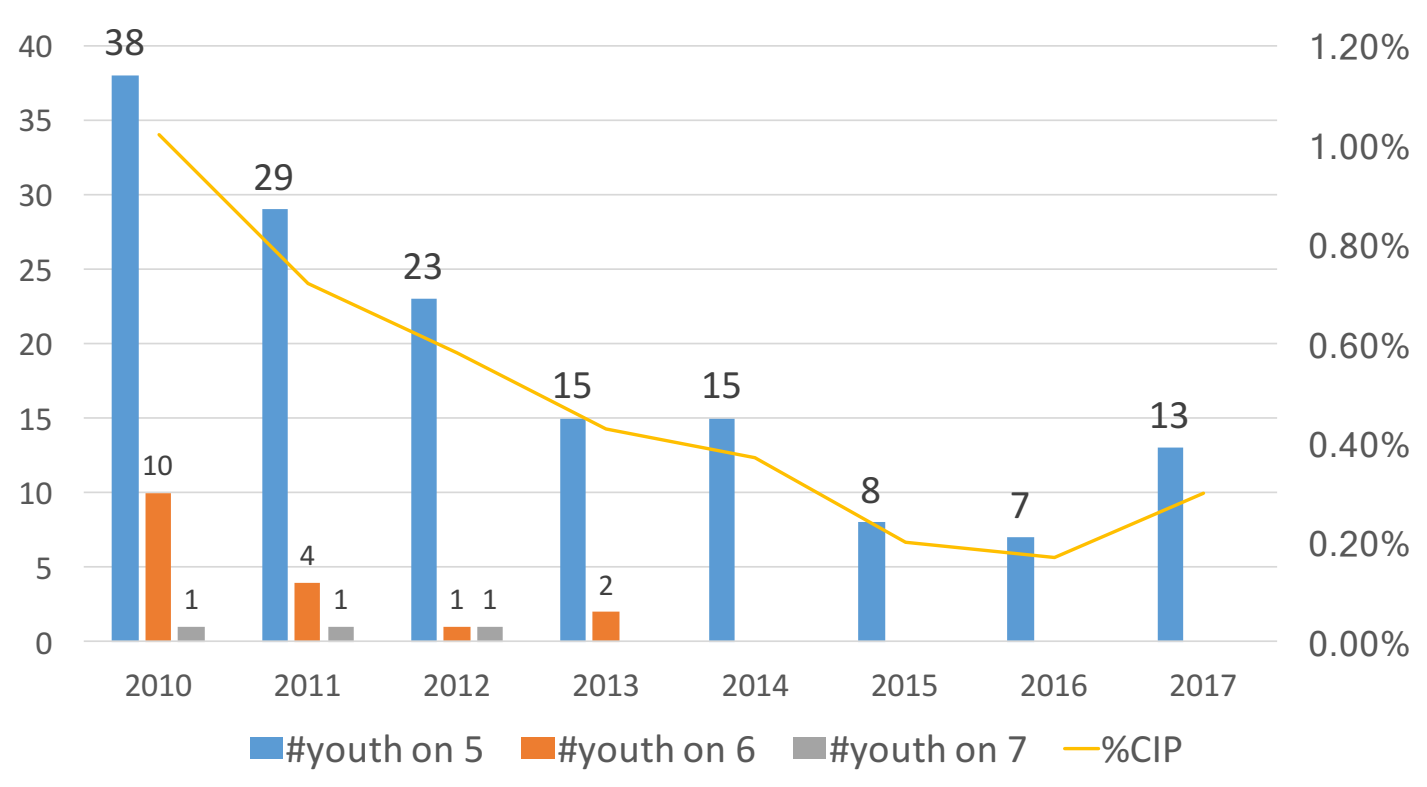

References:

1. Zito J et al. Pediatrics. 2008; 121; e157

2. DosReis S et al. Pediatrics. 2011; 128; e1459

3. Brenner $S$ et al. J Child Fam Studies. 2014; 23(4): 666-674

4. United States Public Law 110-351 (2008)

5. United States Public Law 112-34 (2011)

6. CT General Statutes 17a-21a (2004) 